\title{
THE ASSESSMENT OF ORTHOPHOTO QUALITY WITH RESPECT TO THE STRUCTURE OF DIGITAL ELEVATION MODEL
}

\author{
M.Modiri a , H.Enayati ${ }^{\text {b }}$ M. Ebrahimikia ${ }^{c, *}$ \\ a Professor at Malek Ashtar University of Technology, Esfahan, Iran- mmodiri@ut.ac.ir \\ ${ }^{b}$ M.s degree of Photogrammtry, Khaje Nasir university Tehran, Iran \\ ${ }^{\mathrm{c}}$ M.s degree of Photogrammtry, The university of Tehran, Iran - moj_ebrahimikia@yahoo.com
}

KEY WORDS: orthophoto, height displacement, orthophoto elongation, Orthophoto precision, Orthophoto quality, DEM structure

\begin{abstract}
:
Orthophoto is an image which is being corrected geometrically so each object has to be situated on the corrected place consequently. Choosing the best DEM structure with respect to the area topographic is the most challenge which has more important role when dealing with rough surfaces displacements in duration of orthophoto procedures. The Lower DEM resolution makes points density lower and makes the procedure faster but cause to decreasing the product precision in compare to choosing the other one. However if a fine resolution DEM cause to very delicate displacement corrections aside of the other benefits but it makes to appear some undesired visualized errors like as elongation error especially in an areas which are hidden with some obstacles and there are lacks of data in an imaging. For preventing of such error in DEM structure calculation and earning the most benefits, we found and execute some solutions. In other word we answered to this question that what DEM resolution is the best for orthophoto production. In the following we have done some tests. First a dense DEM of a topographic area calculated and edited accurately then its density was reduced in some steps gradually. At each stage the root mean square error (RMSE) of interpolated heights of points which were laid in the distance between the corresponding DEMs pixels has been calculated respectively. Two interpolation methods (Nearest neighbour and Bilinear interpolation) have been used in this test. Decreasing the DEMs density or increasing the pixel size made the amounts of errors high and the rate of this changing dependent on the kind of topography directly. So we divided the area into some reasonable topographic classes then calculated our results for each class separately. The result of each strategy compared with each other and presented in both numerical tables and some illustrated images.

Because of the relation between horizontal precision of orthophotos which are existed in the standard producing instruction and the accuracy of the DEM which are mostly related to its density, the suitable resolution for producing different scale orthophotos at each kind of topographic class have been calculated from mentioned methods consequences and shown as a final result.
\end{abstract}




\section{INTRODUCTION}

Orthophotos are one of the most applicable georefrenced products that usually produced based on aerial or satellite images Having. Geographical and radiometrical data together made the high number of Orthophoto usages in prior stages of most engineering plans which have caused to have more attention to both of the geometric and quality aspects. They are produced in different resolution or scales. Its geometrical resolution highly affected by digital elevation model (DEM) and the quality of geometrical corrections like as aerial triangulation (AT) algorithm so they are important factors in orthophoto generations. The error of AT due to some obvious effects on orthophotos like as displacements. In this paper after passing a review of generation of orthophotos and its necessary steps and factors like as georefrencing procedure and digital elevation model (DEM), we have focused on DEM structure influences on the quality and precision of orthophotos. The precision of DEM is related to images scale and resolution, radiometric quality Of images in matching procedure, DEM grid spacing and the way of its producing, Simard(1997). The best amount of DEMs density is one of the largest challenges in orthophoto producing which makes the time of producing low

\section{ORTHOPHOTO PRODUCTION AND DEM IMPORTANCE}

In this session, principles of orthophoto with respect to images orientation and DEM are presented. The importance of using a suitable DEM and effects of making any deformation on it are explained theoretically and practically.

\subsection{Orthophoto}

During of orthophoto production, related images with their orientation and DEM are used. At these process effects of height displacements and tilt angle effects on captured images are removed, (Chapter14,2014). In fact at this way DEM cells whom size are dependent on the orthophoto size are imaged on the vertical datum. DEM pixels are got values from one of interpolation algorithm on gray values of corresponded DEM cells on orthophoto(fig1),(Leica photogrammetry,2008). With the hypothesis that using a correct georefrencing parameters and a correct DEM cause to place each feature on its true position correctly (maximum error of DEM based on the scale of orthophoto would be defined).

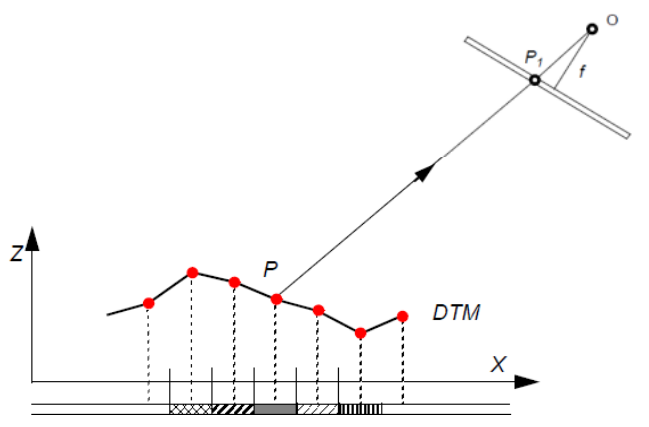

Figure1, getting gray value to rectified image(orthophoto) or high. If decreasing the density makes the procedure faster but cause to reach unreal DEM and incorrect rectification on orthophotos either incorrect position of features and relative displacements between same features on coverage images. At the next part of this paper we have a review on how the orthophoto is made based on corresponded DEM and images orientation factors as well as the effect of DEMs density on horizontal displacements of orthophotos. For explaining more about the effects of applying a suitable DEM structure on removing of the most height differences of captured images, we have tried to apply some unreasonable resolution of digital elevation model (DEM) in orthophoto producing and results of them have shown respectively. In the third part, the permissible amount of orthophoto error is expressed then with respect to the importance of DEM precision, the best amount of DEMs density estimated for each kind of topography and scales of orthophotos.In the result part, outputs of previous sessions summarized and reviewed totally then a table about the suitable amount of DEMs density for each scale of orthophoto at each kind of topographic classes is presented.

\subsection{Shape and precision of DEM at orthophoto production}

At this part, the effect of DEM and imaging configure on orthophoto are studied. For assessing how much they are related to each other,effect of any changes on DEMis illustrated on orthophoto and calculated consequently.

\subsubsection{DEM height changes on image rectification}

In fig (2), point 1 on a captured image is an image of a place which is higher than its surrounded area. This cause to make some height displacements at orthophoto production. The true place of that point relied on 1'. During of rectifying, using a true DEM, all matched points are respective to each other like as point 1 on the captured image and point1 on DEM. The image of this point on Height Datum is point 1' which its gray value gets back on captured image base on an interpolation algorithm. Next, this DEM was changed and smoothed, fig(2).

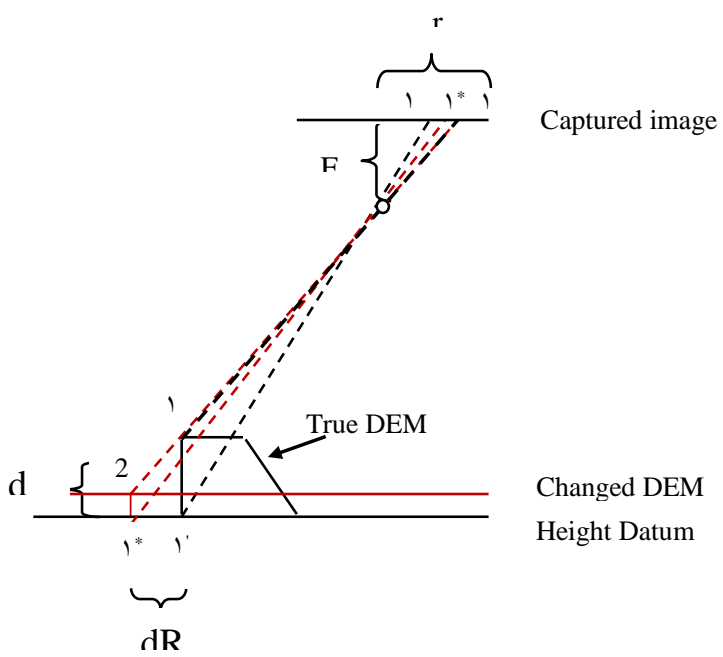

Figure2, The effect of changing DEM on orthophoto production 
Now point 2 on this model is replaced to point 1 and imaged on a different place point $1 *$ instead of the true point 1 ' on orthophoto With supposing that the distance between $1^{\prime}$ and $1^{*}$ as $\mathrm{dR}$, DEM height difference as $\mathrm{dh}$, focal length as $\mathrm{F}$ and the distance between a point on a captured image to image center as r, fig(2); Eq2 shows the relation between DEM points height changes and coordinate displacements.

$$
\begin{aligned}
& \frac{d h}{d R}=\frac{F}{r} \\
& d R=\frac{r d h}{F}
\end{aligned}
$$

With the hypothesis that the focal length is fixed any changes at DEM features height in the corner of image lead to more displacements in compare to those which are near to image center.

\subsection{2 the effect of DEM height changes on orthophoto mosaic}

At this session the effect of DEM height changes on orthophoto mosaic continuously is studied. In fig(3),points1 and 1' are two corresponded points on photos left and right. They are related to a height place(point1 on DEM) which point2 is its base. During of orthophto production with using a true DEM, both of points have to move to point2 (image of the base of the feature). if DEM is changed like what was shown in fig(3), the place of intersection of those points $\left(1,1^{\prime}\right)$ with changed DEM moved to two different places(1 and 1') on orthophoto which cause to make a difference $\mathrm{dR}$ between two same features on orthophoto mosaic.

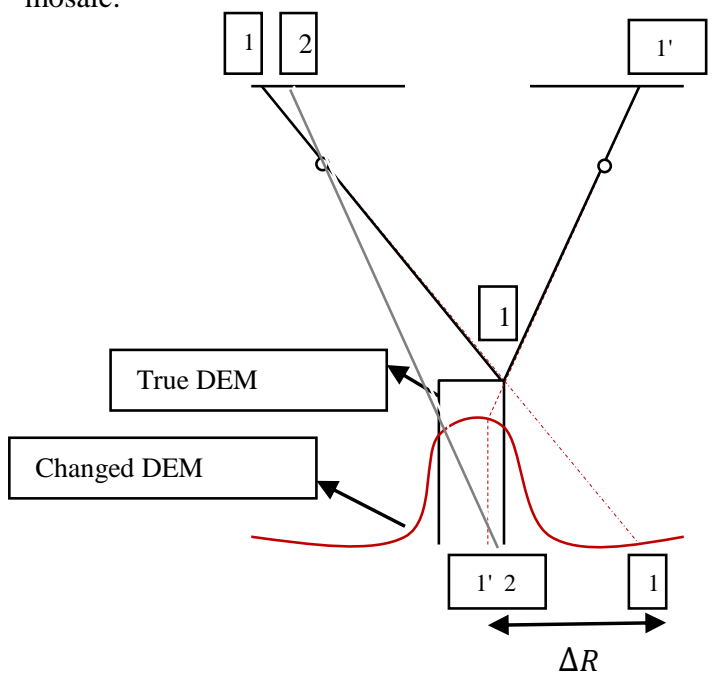

Figure3, The effect of changing DEM on orthophoto mosaic

\section{3 visually study of DEM changes on orthophoto}

For explaining better how much changing DEM could change the coordinate of orthophoto features, an orthophoto mosaic sheet is presented which includes some clear elongation displacements. This area located in 10 to 20 percent of image coverage and contains high height differences, fig (4).

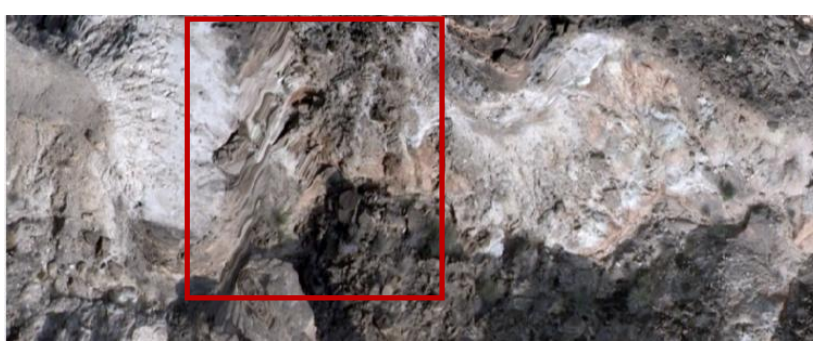

Figure4, Part of first orthophoto

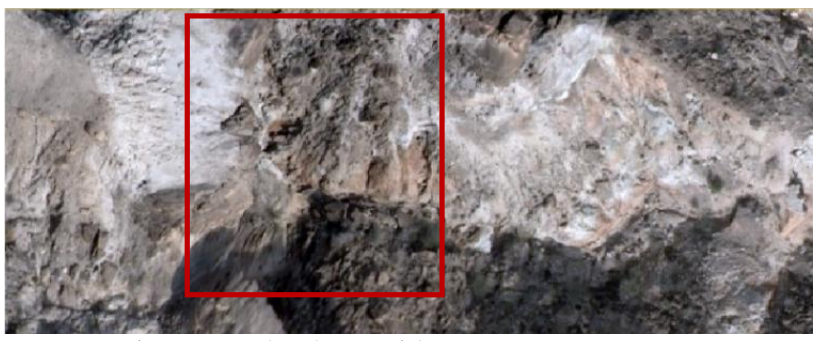

Figure5, orthophoto with sparser DEM(15 m)

Differences of highest to lowest place are about 100 meters in 1.4 meter of distance, so using a suitable DEM is an important factor. The existed elongation in this image is because of the lack of data in the captured image which was used in this orthophoto. Fig(5) shows another orthophoto of this area which is produced with a smoother DEM. The density of used DEMwas decreased in fig (5) so many places in fig(4) is remaind originally without any rectifying process on it. With comparison of two figures $(4,5)$, any differences on DEM because of its density cause to some clear changes on orthophoto. The coordinate of the shown point on the first orthophoto(fig(4)) is 671881.57,3020168.72, DEM value at this point is 68.15 meter and on the other orthophoto(fig(5)) with sparser DEM density is 671883.16,3020171.37 and its DEM value is 51.57 meter. The distance of this point from the center of the image is 18.87 millimeter and with considering eq2, the difference of displacement on two orthophotos is calculated as 3.085 meter.

$$
\frac{18.87 *(68.15-51.57)}{101.4}=3.085
$$

\section{THE SUITABLE DEM DENSITY}

Digital elevation model is an important factor in producing orthophoto which its precision has a direct influence on orthophoto quality. Amount of this depends on the rate of the area topographic and cause to more error on orthophoto at height places in compare to flats (eq(2)). So attention to DEM density with respect to the type of area is very important. At this session, the suitable DEM density is accessed. First a precise DEM of the area is chosen then the density of it is decreased continuously. At each step height values of all first points are calculated based on two current interpolation algorithms and compared with the first ones. With respect to the orthophto scale, relation between possible horizontal error in orthophoto and height DEM error, amount of possible DEM error and its density would be reached

\subsection{DEM accuracy on orthophoto with respect to}

For defining maximum effect of DEM on orthophoto, Eq(2) is studied again. $\mathrm{Eq}(2)$ can transform to $\mathrm{Eq}(3)$ If entire of image has been used in orthophoto producing and want to estimate the maximum DEM effect on orthophoto producing with supposing of using ultracam camera, $\mathrm{f}=101.4, \mathrm{D}=61.78 \mathrm{~mm}$ (D: 
maximum Radial distance from image center) With assuming the correct matching, incorrect amount of DEM resolution without respect to topographic type is related to dh directly. So choosing any incorrect DEM pixel size cause to make high differences between calculated and real height values and $\mathrm{dR}$ error on orthophoto.

$$
d R=0.61 \mathrm{dh}
$$

Eq(4) shows the total amount of error in orthophoto in different stage of production.

(Total Orthophoto RMSE)2 = (Triangulation RMSE) $2+($ RMSE of DEM )2

For calculating $\mathrm{dR}$, the amount of average triangulation calculation error is subtracted from the total error of each orthophoto, eq(5).

$\mathrm{dR}=$

$\sqrt{(\cdot .3 * \cdot . . \cdot 1 * \text { Scalemap })^{2}-(1 / 3 * \cdot .3 * \cdot . . .1 * \text { Scalemap })^{2}}$

The error of DEM on orthophoto is calculated base on Eq(4) and supposing that the average differences of features on orthophoto mustn't be more than $0.3 \mathrm{~mm}$ in map scale as well as the triangulation error is about $1 / 3$ of the total error.

\begin{tabular}{|c|c|c|c|}
\hline $\begin{array}{c}\text { RMSE of ultracam } \\
\text { D } \\
\mathbf{d R = 0 . 6 1 d h}\end{array}$ & $\begin{array}{c}\text { RMSE of } \\
\text { DEM(m) }\end{array}$ & $\begin{array}{c}\text { Total } \\
\text { RMSE(m) }\end{array}$ & Scale \\
\hline 0.46 & $\cdot$, $\wedge$ & 0.3 & $1: 1000$ \\
\hline 0.92 & 0.56 & 0.6 & $1: 2000$ \\
\hline 2.3 & 1.4 & 1.5 & $1: 5000$ \\
\hline
\end{tabular}

Table1. Amount of permissible DEM height error in orthophoto production based on eq $(3,5)$

\subsection{Case study and data preparation}

The study area comprises 44940 pixels (DEM values) of flat to too high topographic area, for doing the interpolation calculation in different densities, this area is divided to some same size patterns then the height values of points inside of each of them are calculated based on two interpolation algorithm(Nearest Neighbor and Bilinear). The size of patterns grows after each step with a pixel and the error values as well as averaging of errors (RMSE) are calculated separately. Fig (6) shows area DEM and fig (7) shows area slope map.
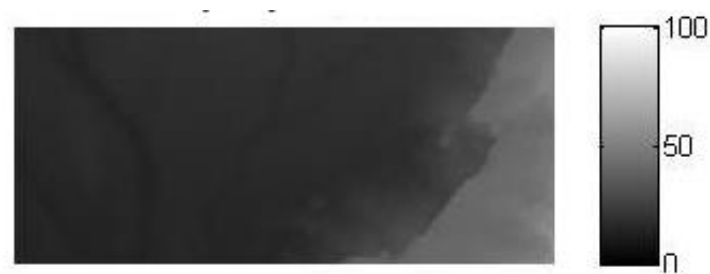

Figure6, Case study DEM(m)
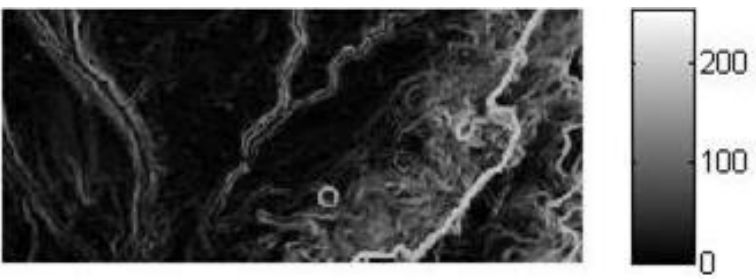

Figure7, Slope map (percent)

Because of the interpolation error is highly dependent to area topographic type, the area is divided to some topographic classes and the average error is calculated for each group separately. Topographic classes are grouped as:
1- More than 70 degree of slope
$2-\quad 50<$ Slope degree $<70$
3 - $\quad 35<$ Slope degree $<50$
4- Slope degree $<35$

These classes are grouped for high slope cliffs, high slope mountains, hills and flat areas. Because of the high topographic area includes a few pixels and are important in true orthophoto production, puts away of calculations. Fig (8) shows classified study area based on defined classes.

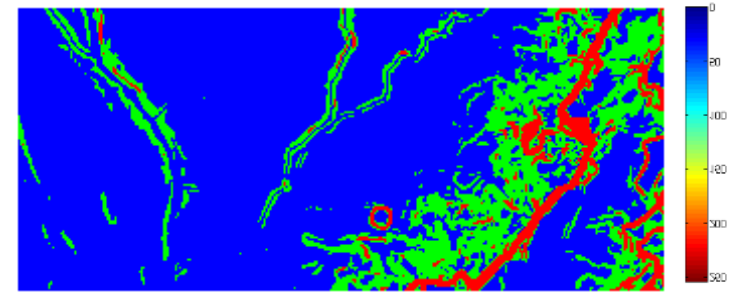

Figure8, Classified slope map based on mentioned classes

At this classifing, pixels in groups 2,3 and 4 are colored with red, green and blue separately.

Total area pixels are 44940, pixels of second class are 2554, pixels of third class are 8621 and there are 33765 pixels in fourth class.

\subsection{Interpolation}

At this session the result of each interpolation is presented.

\subsubsection{Bilinear interpolation results}

The result of precision for Bilinear interpolation on classes 2 to 4 is presented in table 2 .

\begin{tabular}{|c|c|c|c|c|}
\hline \multicolumn{5}{|c|}{ Bilinear Interpolation } \\
\hline $\begin{array}{l}\text { Interpolati } \\
\text { on } \\
\text { resolution } \\
\text { (m) }\end{array}$ & $\begin{array}{c}\text { RMSE( } \\
\text { m) } \\
\text { General } \\
\text { 44940pi } \\
\text { x }\end{array}$ & $\begin{array}{c}\text { RMSE( } \\
\text { m) } \\
\text { Class2 } \\
\text { 2554pix }\end{array}$ & $\begin{array}{c}\text { RMSE( } \\
\text { m) } \\
\text { Class3 } \\
\text { 8621pix }\end{array}$ & $\begin{array}{c}\text { RMSE( } \\
\text { m) } \\
\text { Class4 } \\
\text { 33765pi } \\
\text { x }\end{array}$ \\
\hline 1 & 0.12828 & 0.43572 & 0.08612 & 0.04477 \\
\hline 1.5 & 0.22408 & 0.70805 & 0.15729 & 0.08451 \\
\hline 2 & 0.31824 & 0.96429 & 0.23404 & 0.12912 \\
\hline 2.5 & 0.38878 & 1.12415 & 0.31575 & 0.17490 \\
\hline 3 & 0.46914 & 1.30385 & 0.42683 & 0.20867 \\
\hline 3.5 & 0.50801 & 1.33846 & 0.48095 & 0.25616 \\
\hline 4 & 0.56093 & 1.42665 & 0.56372 & 0.28704 \\
\hline 4.5 & 0.60273 & 1.48178 & 0.62108 & 0.32145 \\
\hline 5 & 0.69539 & 1.67113 & 0.70094 & 0.39907 \\
\hline
\end{tabular}


Table2. Bilinear interpolation precision at each of classes with increasing step of a pixel

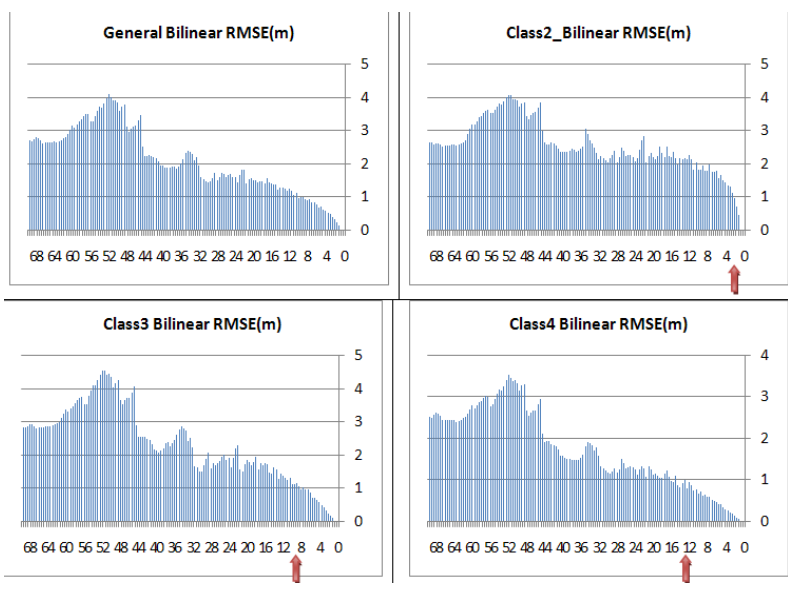

Figure9, Plots of precision changes in Bilinear interpolation in each increasing step. Arrows show the maximum bound of reasonable relation between grid spacing and errors (Horizontal axis: DEM grid spacing, vertical axis: amount of errors)

\subsubsection{Nearest Neighbor Results}

Like as previous method the result of precision for Nearest neighbor interpolation on classes 2 to 4 is presented in table 3 .

\begin{tabular}{|c|c|c|c|c|}
\hline \multicolumn{5}{|c|}{ Nearest Neighbor Interpolation } \\
\hline $\begin{array}{l}\text { Interpolati } \\
\text { on } \\
\text { resolution } \\
\text { (m) }\end{array}$ & $\begin{array}{l}\text { RMSE( } \\
\text { m) } \\
\text { General } \\
\text { 44940pi } \\
\text { x }\end{array}$ & $\begin{array}{c}\text { RMSE( } \\
\text { m) } \\
\text { Class2 } \\
\text { 2554pix }\end{array}$ & $\begin{array}{c}\text { RMSE( } \\
\text { m) } \\
\text { Class3 } \\
\text { 8621pix }\end{array}$ & $\begin{array}{c}\text { RMSE( } \\
\text { m) } \\
\text { Class4 } \\
\text { 33765pi } \\
x\end{array}$ \\
\hline 1 & 0.12829 & 0.43572 & 0.08612 & 0.04477 \\
\hline 1.5 & 0.36895 & 1.23047 & 0.23071 & 0.09606 \\
\hline 2 & 0.38768 & 1.23650 & 0.25972 & 0.12274 \\
\hline 2.5 & 0.60911 & 1.91764 & 0.44941 & 0.20107 \\
\hline 3 & 0.61604 & 1.88487 & 0.48431 & 0.21380 \\
\hline 3.5 & 0.77334 & 2.23574 & 0.71578 & 0.30074 \\
\hline 4 & 0.76831 & 2.19252 & 0.70815 & 0.32078 \\
\hline 4.5 & 0.90864 & 2.44190 & 0.89602 & 0.40934 \\
\hline 5 & 0.95253 & 2.54670 & 0.92748 & 0.44767 \\
\hline
\end{tabular}

Table3. Nearest neighbor interpolation precision at each of classes with increasing step of a pixel

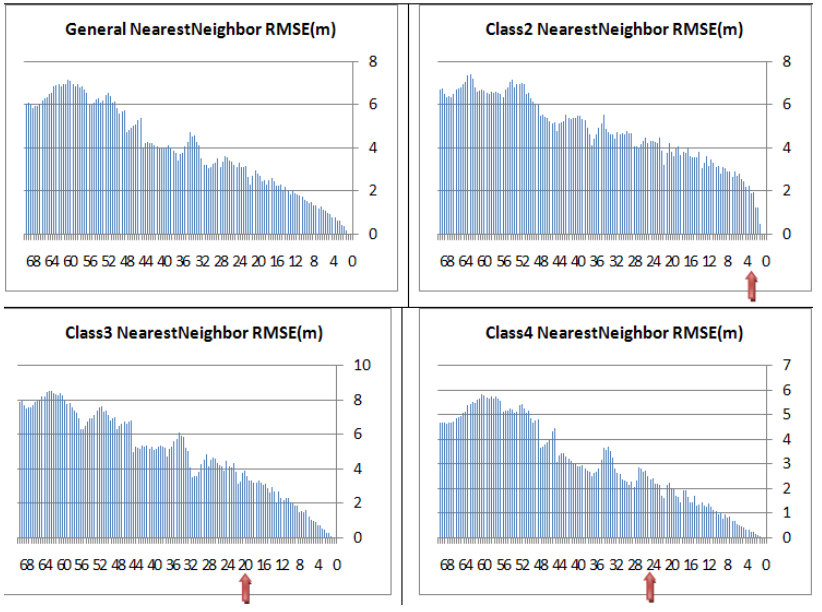

Figure10, Plots of precision changes in Nearest neighbor interpolation in each increasing step. Arrows show the maximum bound of reasonable relation between grid spacing and errors(Horizontal axis: DEM grid spacing, vertical axis: amount of errors)

\section{RESULTS}

The precision of rothophotos like as the other geometrical product are dependent on the used data in duration of production.

DEM and the procedure quality are two important factors in orthophoto production. DEM cause to correct height displacements and the quality and the precision of it affect orthophotos directly. The height of each pixel must calculate based on an interpolation algorithm on some related DEM height values. At this paper, orthophoto production based on captured images, images orientation and DEM was studied and geometric equations and mathematical models precisely explained. The role of DEM was carefully took attention with choosing a sheet of orthophoto which included some elongation errors and the effect of its changes with DEM was studied. With respect to DEM effects on orthophoto feature coordinate displacements and eq $(1,2)$, amount of decreasing of density must be done with respect to map scale carefully. In the other test, the relation between the precision of interpolated height values, the density, and interpolation algorithm and slope rate changes has been done. At this test a precise dense DEM of an almost high topographic area prepared and at some steps its density has been decreased. The amount of differences between calculated and real height values have been assessed at each topographic classes and algorithms. Taking a review of bilinear algorithm results in table2 shows that increasing DEM pixel size lead to reducing amount of interpolation precision. This manner continues in all plots of fig(9) until DEM density is more sparse and there isn't any reasonable relation between them. In fig(9), the plot of area without attention to topographic classes shows error values (vertical axis) to interpolation distance (horizontal axis) in a curve line but its increasing manner until $26 \mathrm{~m}$ of resolution is clear. However because of this plot considers all of topographic classes could be much important in decision. Other plots at this figure show reasonable manners for classes of 2 to 3 . In plot of class 2 , the linear manner was removed and took a cure shape so faster than other classes. This form shows high sensivity of this class to decreasing the DEM density. At plot of the other classes $(3,4)$, this manner can be seen but the rate of changes is slower and 
the linear manner has been kept until larger DEM pixel size. All plots show that the manner of changes hasn't been reasonable at the end tail of figures and we can't consider these values in our results. Both interpolation algorithm results (Nearest neighbor and bilinear) have the same manner and error values in nearest neighbor are larger and stricter than the other. However choosing DEM large cells cause to decrease the height meaning relation between the grid points isn't possible and proposed to fixing this bound value for larger orthophoto scales. In fig(11), error values at each interpolation algorithms at each DEM grid space are shown. Nearest neighbor algorithm shows higher error values in steeper areas and the discontinuously is clear between its output values whereas Bilinear interpolation outputs because of interpolating in two dimensions are smoother and the shape of area in estimating the errors with increasing the grid space is kept.

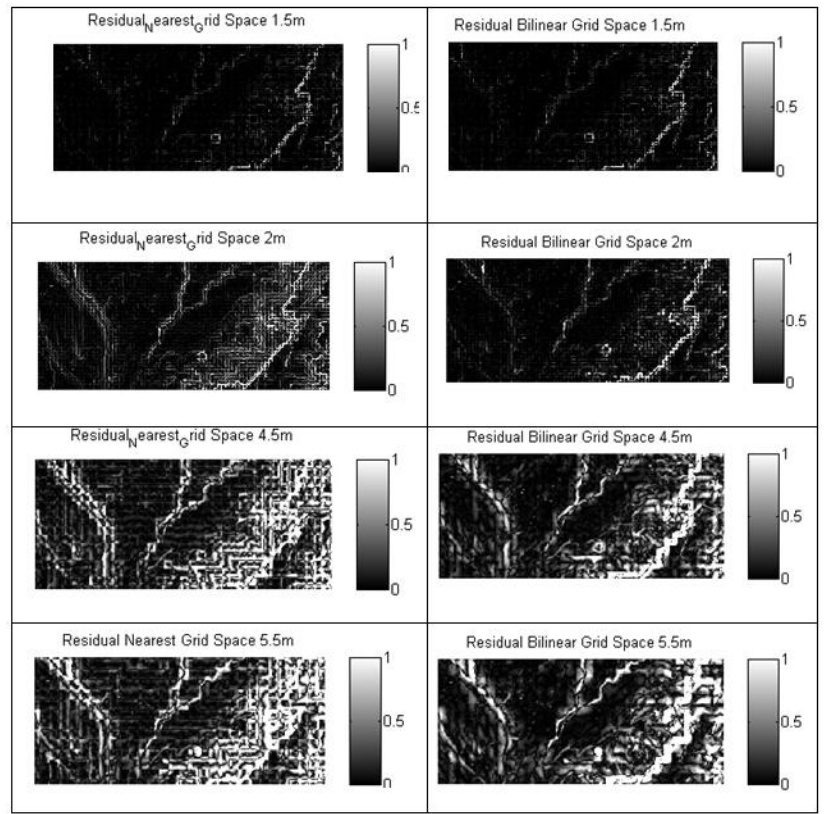

Figure11, Error values on DEM pixels at each interpolation algorithm(Left plot: Nearest neighbor interpolation, Right plot: Bilinear interpolation)

With comparison of both of two interpolation algorithms and error plots, maximum possible errors at each scale of orthophotos in case of using Ultracam images has been extracted and presented in table(4) consequently.

\begin{tabular}{|c|c|c|c|c|}
\hline \multicolumn{3}{|c|}{$\begin{array}{l}\text { DEM Resolution(m)with respect } \\
\text { to each topographic class }\end{array}$} & \multirow{2}{*}{$\begin{array}{c}\text { Maximu } \\
\text { m } \\
\text { Possible } \\
\text { Error(me } \\
\text { ter) }\end{array}$} & \multirow{2}{*}{$\begin{array}{c}\text { Orthoph } \\
\text { oto } \\
\text { Scale }\end{array}$} \\
\hline $\begin{array}{c}\text { Topogra } \\
\text { phic } \\
\text { Class1 }\end{array}$ & $\begin{array}{c}\text { Topogra } \\
\text { phic } \\
\text { rClass }\end{array}$ & $\begin{array}{c}\text { Topogra } \\
\text { phic } \\
\text { Class3 }\end{array}$ & & \\
\hline 1 & $r$ & $\Delta, \Delta$ & $\cdot, \uparrow 9$ & $1: 1 \ldots$ \\
\hline 1,0 & $\Delta, \Delta$ & $9, \Delta$ & $\cdot, 94$ & $1: \Gamma \cdots$ \\
\hline i & 11,0 & $r \xi, \Delta$ & $r, r$ & $1: \Delta \cdots$ \\
\hline i & $11, \Delta$ & $T F, \Delta$ & $f, \xi$ & $1: 1 \ldots$ \\
\hline
\end{tabular}

Table2. Calculated suitable DEM resolution for each scale of Orthophotos

\section{REFERENCES}

BC Ortho-Image specification;Base mapping and cadastre,GeoBC; March2011.

Chapter14: Orthophotography-Gis-Lab.gis-

Lab.info/docs/books/aerial-mapping/cr1557_14.pdf.Access $10 / 2014$.

Degaard Nielsen.M, “True orthophoto generation”, IMM-

Thesis;Technical university of Denmark.

[8] Report to the U.S. Geological Survey on Digital

Orthoimagery, ASPRS, 2005,

http://nationalmap.gov/report/ASPRS_Report_on_Digital_Orth oimagery.pdf

"Guidelines for Best Practise and Quality Checking of Ortho Imagery", European Commission, Directorate General, Joint Research Centre, Institute for the Protection and Security of the Citizen, Monitoring Agriculture with Remote Sensing

Unit.Issue 2.3,(2004). http://marsunit.jrc.it/Mapping/.

Honkavaara.E, Markelin.L, Marttinen.J and Vilander.M, "External Quality Control of Medium-scale Orthophoto Production", case Finnish Land Parcel Identification System' Nordic Journal of Surveying and Real Estate Research , 2004,VOL 1, p131:P143.

Honkavaara.E, Kaartinen.H, Kuittinen.R, , Huttunen.A, and Jaakkola.J "Quality of FLPIS Orthophotos", Reports of the Finnish Geodetic Institute, (1999). 99:1.

Leica photogrammetry suiteproject manager;users guide February 2008.

Pala.V, Arbiol.R "True Orthoimage Generation in urban areas, Institute cartographic of catalunya;P309:P314.

Simard.G "Accuracy of digital orthophotos", A report submitted for the degrss of masters in engineering; the university of New Brunswick; February 1997. 